\title{
Inbreeding, self-fertilization, lethal genes and genomic coalescence
}

\author{
S. H. JAMES \\ Botany Department, The University of Western Australia, Nedlands, Western Australia, 6009
}

\begin{abstract}
A deterministic computer model was used to investigate the elevation in frequency of coalesced genomic heterozygotes, i.e. heterozygotes with reduced numbers of independently assorting supergenic units at meiosis, in inbred populations in which every supergenic unit carried a recessive lethal gene. Inbreeding was manipulated in terms of $s$, the proportion of self-fertilizations per generation, and $F$, Wright's Inbreeding Coefficient which measures the degree of relatedness between individuals within a population. It was found that where $F$ was greater than about 0.19 , coalesced heterozygotes were lifted to high frequencies ( $>50$ per cent) in the population in the absence of self-fertilization. Coalesced heterozygotes were lifted to high frequencies in populations with $F$ less than 0.19 by appropriate levels of self-fertilization. Populations with fewer independently segregating supergenic units at meiosis were more sensitive to the effects of inbreeding than those with larger numbers of such units. The possible significance of these observations in terms of the evolution of genetic systems and in conservation practice is briefly discussed.
\end{abstract}

Keywords: evolution, genomic coalescence, inbreeding, lethal genes, permanent hybridity, selfpollination.

\section{Introduction}

Genomic coalescence may be defined as the evolutionary rearrangement of the genotype in diploid sexual organisms into fewer linkage groups or into linkage groups which exhibit reduced levels of recombination. It is supergene formation and may be reflected by a reduction in chromosome number, by structural hybridity, by the alteration of position and frequency of chiasmata, by epistatic linkage in the formation of coadapted gene complexes, and by devices which promote the polarized segregation of chromosomes at meiosis. Because related taxa often differ with respect to chromosome structure and other attributes modulating recombination, genomic coalescence may be a common outcome of micro-evolutionary processes. If, however, genomic coalescence reduces the recombinational capability of its products, it may thereby expose them to an increased risk of extinction. Lineages which avoid genomic coalescence should maintain their recombinational potential and the evolutionary capability generated by that potential; they are more likely to be successful as ancestors of future generations. While the advantages of free recombination may account for the long-term evolutionary success of lineages which avoid genomic coalescence, there must be advantages associated with coalesced genomes that are visible to natural selection in the short-term, and which tend to drive lineages into risk and extinction.

The natural tendency for linkage to increase was first noted by Fisher (1930) and the phenomenon has been the centre of considerable discussion since. A variety of theoretical situations have been described in which the tendency may be promoted or countered (e.g. Turner, 1967; Maynard Smith, 1977), but the discussion has not examined extreme situations in which coalesced genomes are clearly advantageous. Of particular interest is the role of linkage in inbred populations carrying high frequencies of recessive lethal genes.

It is commonly held that the equilibrium frequencies of deleterious alleles, such as recessive lethals, will be lower in inbreeding populations than in outcrossing populations. Charlesworth et al. (1990), in agreement with Lande \& Schemske (1985), contend that 'this conclusion is quite correct for recessive lethals and sublethals'. Indeed, it is the expectation arising from classical population genetic theory which demonstates that recessive lethal genes will be reduced in frequencies to levels maintained by mutation pressure. However, deleterious recessives and lethal genes are common in many plant populations. Substantial levels 
of seed abortion resulting from recessive lethal polymorphisms in natural populations characterize many diploid sexual plants (Sorensen, 1969; Ledig, 1986; Weins et al., 1987; Burbidge \& James, 1991). It appears, however, that the phenomenon is often ignored. Because plants exhibiting very low levels of seed set following self-pollination would need to be heterozygous for quite large numbers of lethals if the incompatibility was postzygotic, the incompatibility is often presumed to be prezygotic (e.g. see Kendrick \& Knox, 1989). Standard population genetic theory, which indicates that natural selection would reduce the frequency of any recessive lethal gene to very low levels, may well be valid in panmictic systems where the individual recessive lethal genes may be so diluted in the population as to be essentially invisible. With self-fertilization, however, the probability of individually rare recessive lethals becoming associated as homozygotes is elevated. With very large numbers of loci polymorphic for recessive lethals, and with high levels of inbreeding, only a small proportion of the zygotes produced may be viable. This would place intense positive selection pressure on devices minimizing the proportion of inviable zygotes produced. Plants may respond by coalescing the genome. This may ultimately concentrate the genome into two supergenic alleles held in permanent hybridity, thereby reducing the frequency of inviable zygotes to 50 per cent as, for example, in autogamic complex hybrids such as Oenothera lamarckiana and Isotoma petraea, which are maintained by balanced post-zygotic lethal systems.

In Isotoma petraea, a member of family Lobeliaceae (Angiospermae), the ability to cross-pollinate is dependent upon the protrusion of the stigma from the anther tube. The frequency of flowers with retained stigmas thus provides a minimum estimate of the frequency of self-pollination within plants. In some populations, this frequency approaches 100 per cent, permitting the analysis of population genetic architecture under conditions of very high inbreeding levels. These populations exhibit elevated levels of genetic heterozygosity, polymorphism for seed aborting recessive lethal genes, and have evolved complex hybridity.

Recently, two deterministic models purporting to simulate the fixation of a doubly interchanged genome as nascent 06 complex hybrids in a population of Isotoma petraea have been described (James et al., 1990). The models recognized seven independently segregating supergenic loci in the ancestral forms corresponding to seven pairs of homologous chromosomes which exhibited terminal chiasma localization. Three of the erstwhile independently assorting supergenes were combined by interchange into a ring-of-six
(06) association. The 06 consisted of three interchanged chromosomes forming an $N$ complex and the three homologous chromosomes with primitive structure forming the complementary $S$ complex. Thus, in the N.S 06 hybrids, the number of independently segregating units was reduced to five and the genome may be considered to have been coalesced from seven to five. The models also recognized a reduced fecundity ( 73 per cent) in the 06 due to their irregular behaviour at meiosis which would be significant in determining the relative fertility of the heterozygotes as seed parents. The models assumed that all supergenic alleles were recessively lethal, that is, each chromosome was considered to carry one or more mutations that would prevent its homozygote from contributing to the next generation. It was also assumed that all products of crosses, apart from those homozygous for the interchanged chromosome arrangement $N$, were viable; that is, apart from the interchanged ring-of-six, the value of $F$ (Wright's Inbreeding Coefficient) at all supergenic loci was 0.0 . The two models differed in that in one case, the $N$ complex was fully transmitted in both pollen and embryosac while in the second, the $N$ complex was pollen non-transmissible. It was found that both models elevated the frequency of the 06 interchange heterozygotes in the population to high levels where the frequency of self-pollination, $s$, was very high ( $>97$ per cent).

It may be anticipated that recurrent self-pollination may elevate the frequency of the 06 even more effectively in more inbred populations, that is, in populations characterized by $F$ values greater than 0.0 . In order to examine the effect of the levels of inbreeding in terms of $F$ and $s$ on genomic coalescence conjointly, the nascent complex hybridity model, with free transmission of $N$ in both pollen and embryosacs, is generalized in this paper.

\section{Description of the model}

The model is illustrated in Table 1. $x$ is a genome composed of $n$ independently assorting supergenic units. $n$ is visualized as approximating the haploid chromosome number plus the mean number of chiasmata per cell that are not terminally localized; it approximates the 'recombination index' (Darlington, 1958). $N$ is a derivative genome which reduces the number of independently assorting units in $N . x$ heterozygotes to $m$. N is of monophyletic origin in the population so that all alleles of $N$ are identical by descent. Each chromosome is recessively lethal. Only those selfed progeny heterozygous for all $n$ or $m$ independently assorting units are viable. The frequency of $N . x$ heterozygotes and $x . x$ homozygotes in the adult population is $P$ and $Q$, 
Table 1 Explanation of the population model. See text for detail

\begin{tabular}{|c|c|c|c|c|c|c|c|}
\hline \multicolumn{2}{|c|}{$\begin{array}{l}\text { Female } \\
\text { parent }\end{array}$} & \multirow[b]{2}{*}{$\begin{array}{l}\text { Disjunction } \\
\text { frequency }\end{array}$} & \multirow[b]{2}{*}{$\begin{array}{l}\text { Pollination } \\
\text { type/frequency }\end{array}$} & \multicolumn{2}{|c|}{$\begin{array}{l}\text { Male } \\
\text { parent }\end{array}$} & \multicolumn{2}{|c|}{ Contribution to next generation } \\
\hline Type & Frequency & & & Type & Frequency & N.ax & $a x . a x$ \\
\hline N.x & $P$ & $D$ & $s$ & $\begin{array}{l}N . x \\
N . x\end{array}$ & $\begin{array}{l}1 \\
P\end{array}$ & $\begin{array}{l}(1 / 2)^{m} \\
\frac{1}{2}(1-F)^{m-1}\end{array}$ & $\begin{array}{l}0 \\
\frac{1}{4}(1-F)^{n}\end{array}$ \\
\hline$x . x$ & $Q$ & 1 & $\begin{array}{l}s \\
t\end{array}$ & $\begin{array}{l}x . x \\
x . x \\
N . x\end{array}$ & $\begin{array}{l}Q \\
1 \\
P\end{array}$ & $\begin{array}{l}\frac{1}{2}(1-F)^{m-1} \\
0 \\
\frac{1}{2}(1-F)^{m-1}\end{array}$ & $\begin{array}{l}\frac{1}{2}(1-F)^{m-1}(1-F)^{n-m+1} \\
(1 / 2)^{n} \\
\frac{1}{2}(1-F)^{m-1}(1-F)^{n-m+1}\end{array}$ \\
\hline & & & & $x . x$ & $Q$ & 0 & $(1-F)^{n}$ \\
\hline
\end{tabular}

$x=$ the standard genome with $n$ independently segregating supergenic units.

$x . x=$ the primitive homozygote.

$N=$ the coalescing genome; $N . N$ homozygotes are excluded from the population by zygotic lethality.

$N . x=$ the coalesced heterozygote with $m$ independently assorting supergenic units.

$P=$ the population frequency of $N . x$, and $Q=1-\mathrm{P}$.

$D=$ the relative gametic fertility of the $N . x$ heterozygote as seed parent.

$s=$ the recurrent selfing rate, and $t=1-s$.

All genomic components are freely transmissible through both pollen and embryosacs. For the cases examined in this paper, in which $m=n-1$, the frequency of the $N . x$ heterozygote in the next generation is given by

$$
P^{\prime}=\frac{P s D / 2^{m}+P t D(1-F)^{(m-1)} / 2+P Q t(1-F)^{(m-1)}}{\left[P s D / 2^{m}+P t D(1-F)^{(m-1)} / 2+P Q t(1-F)^{(m-1)}+P^{2} t D(1-F)^{n} / 4+P Q t D(1-F)^{n} / 2+Q s / 2^{n}+P Q t(1-F)^{n} / 2+Q^{2} t(1-F)^{n}\right]}
$$

respectively. Since $N . N$ homozygotes are always inviable, $P+Q=1$. The relative seed fecundity of $x . x$ homozygotes is 1 , and that of $N . x$ heterozygotes $D$. The recurrent selfing rate is $s$. The inbreeding coefficient of the population is $F$ so that the probability of choosing two alleles of a supergene (apart from alleles of $N$ ) carrying identical recessive lethals is $F$. All N.N homozygotes are lethal, whether they be produced from crosses or selfs.

The value of $P^{\prime}$ (the value of $P$ in the next generation) was determined according to the equation given in the legend to Fig. 1.

\section{Results}

The model was run for particular values of $s, F, D, n$ and $m$. Only cases where $m=n-1$ (equivalent to a single interchange or chromosome fusion) are reported here. In any run, the population was started with $P=0.01$ and $Q=0.99$ and cycled across 50 generations. Two series of experiments are reported here.

Results for the first series of experiments are summarized in Fig. 1. The parameters and course of a 50 -generation cycle leading to the elevation of the coalesced heterozygote to a frequency of 0.5 is presented in Fig. 1a. Courses of the 50-generation cycles following stepwise variations of each parameter about the value used in Fig. 1a are presented in Fig. 1b-e. It can be seen that a sufficient level of inbreeding will elevate the frequency of the coalescent genome heterozygotes within the population. The necessary level of inbreeding may be generated by recurrent self-fertilization ( $s$, Fig. 1b), by a sufficiently high level of genetic relatedness between individuals within the population $(F$, Fig. $1 c)$, or by a combination of both. The effectivity of the coalescing device will also be dependent upon the relative fitness of the heterozygote, symbolized in this model by $D$, the relative ovule fecundity or meiotic disjunction frequency (Fig. 1d). The level of inbreeding necessary to elevate a coalesced heterozygote to a particular frequency in a population also decreases as the original number of independently assorting supergenes, $n$, decreases (Fig. 1e), and the level of inbreeding capable of inducing genomic coalescence from $n$ to $n-1$ would induce coalescence from $n-1$ to $n-2$ even more rapidly. Genomic coalescence, therefore, would be a self-reinforcing or orthogenetic tendency.

In the second series of experiments, the level of selffertilization, $s$, necessary to elevate the frequency of a 
(o)

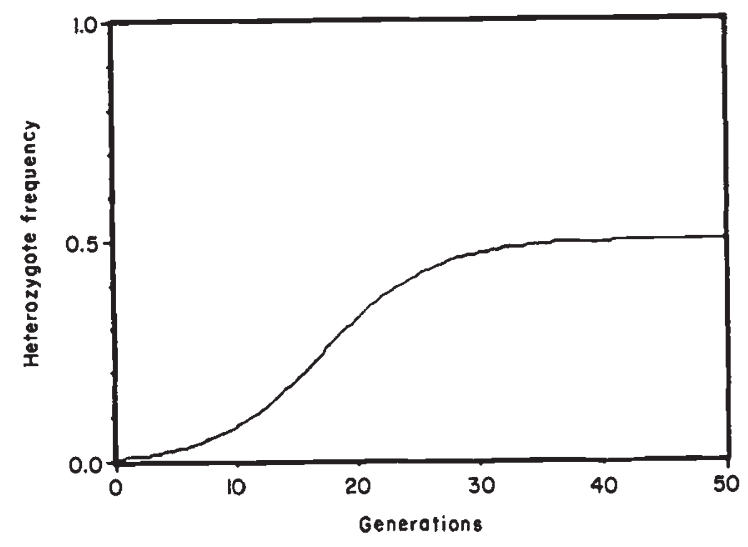

(b)

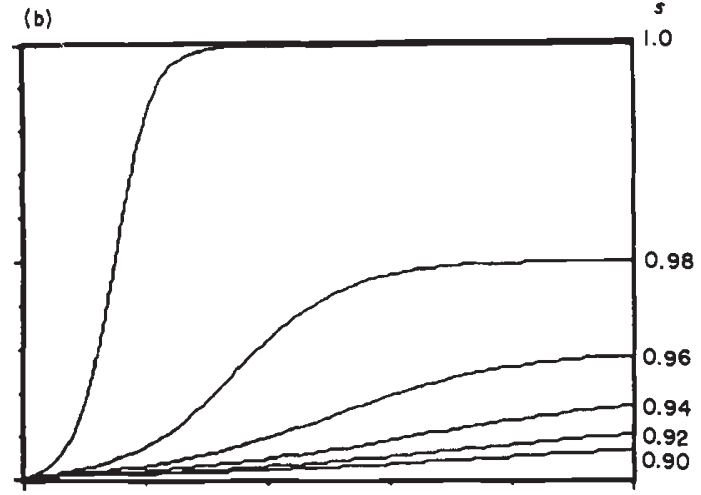

(c)

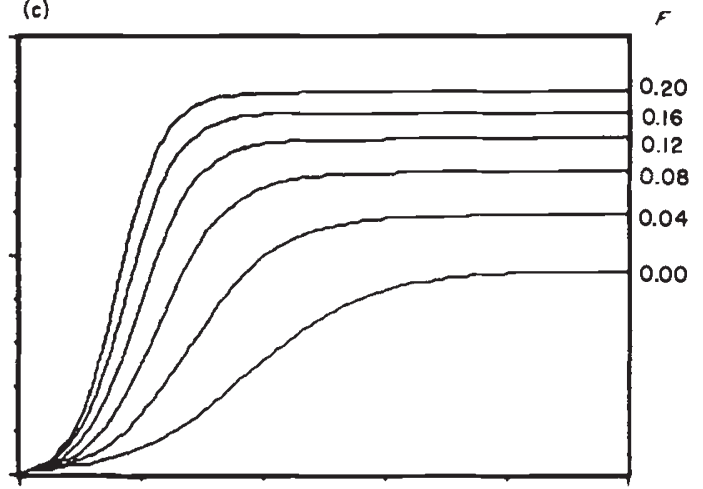

(d)

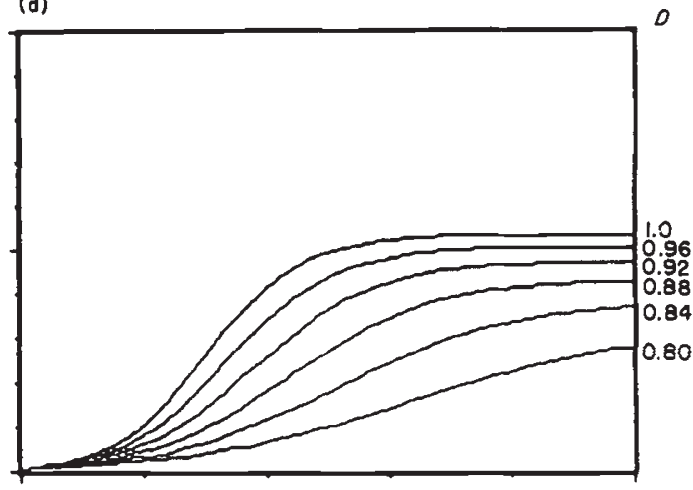

(e)

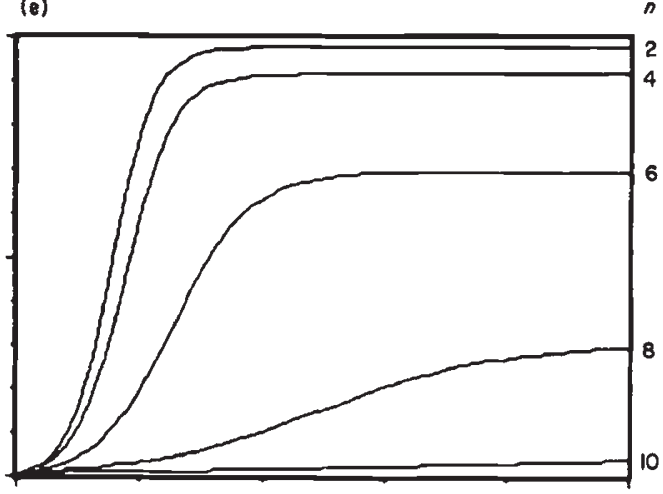

Fig. 1 Coalesced heterozygote frequencies across 50 sequential generations calculated according to the model presented in Table 1. (a) The course of a single model run with the following parameter values. $s=$ recurrent selfing rate $(0.98) ; F=$ inbreeding coefficient of population $(0.01) ; D=$ relative ovule fertility of heterozygote $(0.95) ; n=$ original number of independently assorting supergenic units (7). In these runs, the coalesced heterozygote had $n-1$ independently segregating supergenic units. (b-e) Model runs with one parameter stepped as indicated at right of each figure, the remaining parameters constant, with values as in $(a)$.

coalesced heterozygote to 0.5 for particular values of $F$ within the population, for $D$ as given in Fig. 1a, was determined for a range of $n$, with $m=n-1$. The results of this exercise are presented in Fig. 2. It can be seen that when $F$ is greater than about 0.19 , coalescent heterozygotes will be raised to 0.5 even without any self-fertilization, i.e. under conditions of complete panmixia. With population levels of $F$ below this critical value, high levels of self-fertilization were necessary to elevate the frequency of coalescent heterozygotes above 0.5 , but the population response to self-fertilization was highly dependent upon $n$, the number of independently assorting supergenes. In general, providing $F$ was less than the critical value (about 0.19 ), populations in which $n$ was greater than about 7 were reasonably resistant to self-fertilization. 


\section{Discussion}

The model described in this paper indicates that in a population in which each supergenic allele carries a recessive lethal gene, inbreeding, due either to self-

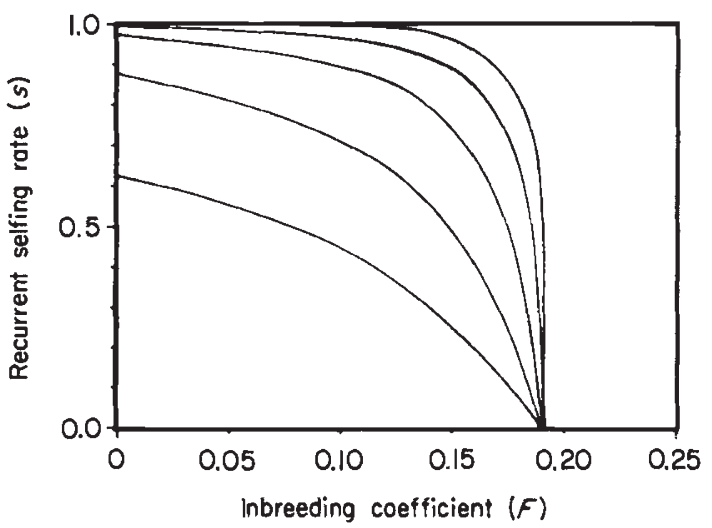

Fig. 2 These graphs describe the levels of recurrent selfing, $s$, and the population inbreeding coefficient, $F$, necessary to elevate the population frequency of coalesced heterozygotes with $n-1$ independently segregating supergenic units relative to the $n$ independently segregating supergenic units of the primitive forms, to 50 per cent, for $n=2$ (lower left), 4, 6 , 8 and 10 (upper right), according to the model described in Table 1.

fertilization $(s)$ or to relatedness of individuals within the population $(F)$, or both, will lead to an increased frequency of coalescent genome heterozygotes in the population. What is modelled then, is the procedure by which autogamy, systems of reduced recombination, and lethal polymorphisms are associated into permanent hybrid genetic systems. If $F$ was greater than about 0.19 , the frequency of coalescent genome heterozygotes would increase even in the absence of self-fertilization. The critical value of $F$, here approximating 0.19 , is obviously directly dependent upon the value of $P$, here set at 0.5 , chosen to define the isopleth; lower values of $F$ and/or $s$ would be required to elevate the frequency of the coalescent genome heterozygotes to levels less than 0.5 .

The model presented maintained $F$ constant over generations that were characterized by constant levels of self-fertilization. However, a constant level of selffertilization would influence $F$, so that an inbreeding equilibrium, in which $F=(1-t) /(1+t)=s /(2-s)$, should be established. Thus, for $s$ greater than about 0.32 , we might expect $F$ to exceed 0.19 and the frequency of coalesced heterozygotes should increase, regardless of subsequent values of $s$. Consequently, the model presented must underestimate the power of inbreeding, in association with high levels of recessive lethal polymorphism, to elevate the frequency of coalescent genome heterozygotes in populations.

Genomic coalescence may be driven into populations having appropriate levels of inbreeding and lethal polymorphism by natural selection because, under the circumstances given, the coalesced heterozygote, $N . x$, may be fitter than the primitive uncoalesced homozygote, $x . x$. On selfing, $x . x$. will produce $1 / 2^{n}$ viable zygotes while $N . x$ will produce $1 / 2^{m} \times D$ viable zygotes, where $D$ is the relative ovule fecundity of $N . x$. Providing that $n$ and $m$ are integars with $n>m$ and that $D$ is more than $1 / 2^{(n-m)} N . x$ will produce more viable zygotes than $x . x$.

Although of short-term adaptive utility in elevating the proportion of viable zygotes above $1 / 2^{n}$ in highly inbreeding populations fraught with recessive lethal polymorphisms, genomic coalescence would reduce the recombinational diversity of the lineage. This, in turn, must reduce the adaptability and long-term evolutionary potential of the lineage and thereby increase its risk of extinction. In addition, the model ignores mutation. Genomic degradation and recurrent mutation of competent genes to deleterious and even lethal mutants is a constant attribute of DNA-based life and is minimized by a variety of DNA repairing mechanisms. Biparental sexual reproduction involving recombination mechanisms provides a system whereby deleterious mutations may be removed from corrupted genomes. Where genomic coalescence is driven into a genetic system already encumbered with high levels of inbreeding and a ubiquity of recessive lethal genes, this aspect of sexual reproduction must be inhibited and the genome must be pushed towards further degradation. Thus, lineages responding positively to selection for genomic coalescence risk decreasing survivability and orthogenetic progress towards extinction.

The tendency towards genomic coalescence, risk and extinction may well provide an engine which drives evolutionary progress by eliminating those lineages which do not, perhaps serendipitously, incorporate adaptations that insulate against the forces of genomic coalescence into their genetic systems. Such adaptations may affect $s, F$ or $n$, they may accommodate the high genetic load through elevated levels of seed production, or they may direct maternal resources preferentially to heterozygous progeny.

Adaptations affecting $s$ include those structural and mechanical modifications of the flower that prevent or modulate the frequency with which self and cross pollen grains arrive at the stigma, and the physiological discrimination between those two types of pollen. Dicliny, including dioecy, and prezygotic self-incompatibility, may represent the most effective systems modifying $s$. Adaptations affecting $F$ include those 
concerned with dispersal, the size and shape of the individuals and their spatial relationships. Adaptations affecting $s$ and $F$ are likely to have significant ecological impacts not solely linked to the properties of genetic systems. In this regard, boundaries between ecological and genetical relevance may be quite obscured and the relevance to population genetics of adaptations with obvious ecological implications may be overlooked. For example, the ephemeral annual species of Stylidium, which tend to occur in dense carpet-like populations of small, relatively fewflowered plants, do not exhibit the genomic coalescence (dysploid chromosome number reduction) that characterizes their larger racemose-inflorescenced perennial congeners (Burbidge \& James, 1991). Ecological and habit differences evidently affect the breeding systems and cytoevolutionary responses of the two groups. Furthermore, well developed adaptations promoting cross-pollination may result from the strong selection pressures that arise from high levels of inbreeding so that the presence of elaborate crosspollination mechanisms in a lineage may indicate high levels of self-pollination or other forms of inbreeding. This logic applies well to triggerplants (James et al., 1991a), the pseudocopulatory orchid Leporella fimbriata (Peakall \& James, 1989) and the birdpollinated Eucalyptus rhodantha (Sampson et al., 1989).

Factors that affect $n$ include interchange hybridity, which ties chromosomes together into a single linkage group, and the dysploid reduction of chromosome number through centromere loss. However, supergene formation by a reduction in chiasma frequency, chiasma localization or other forms of structural hybridity, such as inversion hybridity, may be an equivalent response. Other coalescing devices may include epistatically induced linkage as may be found in internal chromosome segment transposition heterozygotes, polarized segregation at meiosis as has been demonstrated in certain members of the Australian family Epacridaceae tribe Styphelieae (Smith-White, 1948, 1955,1959 ), apomixis, and asexuality. A larger $n$ may be achieved by increasing the chromosome number, by increasing the numbers and randomness in the location of chiasmata at meiosis, by minimizing structural hybridity and by retaining unencumbered classical sexual reproduction in the genetic system.

Polyploidy is a special case where an increase in chromosome number increases genomic redundancy from two, the normal diploid condition, to three, four or more in the polyploid derivatives. Polyploidy reduces the frequency of homozygotes in selfed progeny below the 25 per cent level for each deleterious allele to a level dependent upon the position of the locus in the chromosome, the ploid level, and the fre- quency of allosyndesis at meiosis. Polyploidy may be regarded as an alternative response to conditions otherwise likely to lead to genomic coalescence (see, for example Banyard \& James, 1979).

In a plant heterozygous for deleterious recessives at $n$ independently assorting supergenic loci, the $1 / 2^{n}$ fully heterozygous progeny genotypes expected on selfing may be a small proportion of the zygotes produced. However, the absolute numbers of fully heterozygous progeny genotypes produced may be quite substantial, if the ovule fecundity is sufficiently high. Thus, in orchids which characteristically have thousands of ovules per ovary and minimal amounts of maternal resources invested into individual seeds, seed wastage on self-pollination would not be as disastrous as in species that produce fewer larger seeds. In species with fewer larger seeds, the wastage of maternal resources in the construction of genetically inadequate seed may be minimized by introducing early-acting, seed-aborting, recessive lethal genes into the supergenic alleles. This has apparently occurred in the evolution of the seedaborting lethal systems in the nascent 06 complex heterozygotes of Isotoma petraea at Pigeon Rock (James et al., 1991) and is characteristic of the perennial Stylidium species in Western Australia (Burbidge \& James, 1991). In Eucalyptus camaldulensis only about 7 per cent of the several hundred fertilized ovules per ovary succeed in forming seeds; a large majority succumb to intense competition within the developing capsules. The surviving seeds, however, include an excess of heterozygotes following selfpollination, or a preponderance of outcrosses following open pollination (unpublished results). Thus, postzygotic seed selection systems, based on seedaborting recessive lethal genes or on competition between sibs, may preferentially channel maternal resources to the most adequate progeny genotypes. Such devices would minimize the segregational load associated with high frequencies of deleterious alleles and elevate the fitness of self-pollinating plants.

A positive response to selection for genomic coalescence may require extreme circumstances to be operating in a target population. The very high levels of inbreeding and the ubiquity of recessive lethal factors necessary to elicit the response may seem unreal. However, strong evidence for the process has been adduced from field and experimental observations of complex hybridity in Isotoma petraea (James, 1965, 1970), especially in the Pigeon Rock 06 population in which the genetic system evidently arose (James et al., 1990, 1991b). It has been demonstrated that populations of Stylidium in Western Australia may be highly inbred and polymorphic for seed aborting recessive lethal genes at up to several hundred loci (Burbidge \& James, 1991). The extreme circumstances required to drive 
genomic coalescence may be expected to promote rapid evolutionary responses; failure to elevate the proportion of viable zygotes from $1 / 2^{n}$ may well mean extinction. It is possible that individual hermaphroditic plants, capable of self-fertilization and newly isolated from larger populations, may generate a population in which the extreme circumstances which both require and facilitate genomic coalescence may exist. The important role of self-fertilization in promoting genomic coalescence substantially decreases the relevance of the phenomenon in dioecious species, which includes many plants and most animals, and in plants with pre-zygotic self-incompatibility systems. The fact that it may be largely irrelevant in dioecious populations, combined with anthropocentricity, may well have contributed to the phenomenon, and its causes, being largely overlooked amongst hermphroditic plants capable of self-fertilization. However, intense selection pressures towards genomic coalescence may be expected to be generated in small isolates of any diploid sexual species, and could give rise to dramatic evolutionary episodes indistinguishable from the 'transiliences' described by Templeton (1980a,b).

The elevation of coalesced heterozygotes to high frequencies by inbreeding in populations highly polymorphic for recessive lethal genes provides an important structuring of gene pools. In particular, the average genome in such populations will be composed of a limited number of supergenes and these supergenes will be held at high levels of heterozygosity. The supergenes will contain marker loci, such as allozyme loci, as well as the recessive lethal factors which underpin the polymorphisms. Marker alleles supergenically linked to lethal genes will tend to occur as heterozygotes so that, for that allele, the observed frequency of heterozygotes, $H_{\mathrm{o}}$, may be elevated over expectations, $H_{\mathrm{c}}$. Thus, estimates of $F$, [based on the relationship $\left.F=1-H_{\mathrm{o}} / H_{\mathrm{e}}\right]$ may be reduced and estimates of $t$ [based on the relationship $t=(1-F) /(1+F)$ or on comparisons of parental and progeny genotype arrays] may be enlarged so that the population may appear to be outbreeding. However, elevated $H_{\mathrm{o}}$ may be due to an association with lethal containing supergenes, reduced recombination and inbreeding. Thus, estimates of the breeding systems of inbreeding species, which are based upon the genotype frequencies of parents and progeny arrays derived, for example, from allozyme analyses, are likely to be inaccurate. The primary outcrossing rate, which could be determined from analyses of zygotic genotypes, is likely to differ from the secondary outcrossing rates which are deduced from seed or seedling genotype arrays if substantial levels of postzygotic lethality intervene between fertilization and progeny assay, and the degree of bias towards outbreeding in the estimate may well reflect the intensity of inbreeding in the population.

Outbreeding populations of dioecious organisms and plants equipped with prezygotic self-incompatibility systems would not be immune to genomic coalescence, especialy in populations in which $F$ is greater than about 0.19. As Brown (1979) estimated that the average $F$ amongst outbreeders was of the order of $0.16-0.26$ ( 23 species, compared to 0.75 in seven species of inbreeders), it is possible that many outbreeders are sufficiently inbred through relatedness of individuals within populations so as to elevate coalescent genome heterozygotes to significant frequencies. Again, however, the impact of postzygotic lethality and genomic coalescence on the estimates of $F$ given by Brown (1979), is unclear.

Finally, note that populations which exhibit low levels of allozyme heterozygosity may support the lowest levels of supergene formation and, thereby, the most open recombination systems. Such populations, if they also maintain the highest adaptive and evolutionary capability, may be preferred targets in conservation.

\section{Acknowledgements}

The support of The University of Western Australia and the Australian Research Council is gratefully acknowledged. I thank David Coates, Michelle Waycott, Margaret Byrne, Steve Carstairs and Helen Stace for their comments and discussion.

\section{References}

BANYARD, B. J. AND JAMES, S. H. 1979. Biosystematic studies in the Stylidium crossifolium species complex (Stylidiceae) Aust. J. Bot., 27, 27-37.

BRown, A. H. D. 1979. Enzyme polymorphism in plant populations. Theor. Pop. Biol., 15, 1-42.

BURBIDGE, A. H. AND JAMES, S. H. 1991. Postzygotic seed abortion in the genetic system of Stylidium (Angiospermae: Stylidiaceae). J. Hered., 82, 319-328.

CHARLESWORTH, D., MORGAN, M. T. AND CHARLESWORTH, B. 1990. Inbreeding depression, genetic load, and the evolution of outcrossing rates in a multilocus system with no linkage. Evolution, 44, 1469-1489.

DARLington, C. D. 1958. Evolution of Genetic Systems. Oliver and Boyd, London.

FISHER, R. A. 1930. The Genetical Theory of Natural Selection. Oxford University Press, Oxford.

JAMES, S. H. 1965. Complex hybridity in Isotoma petraea. I. The occurrence of interchange heterozygosity, autogamy and a balanced lethal system. Heredity, 20, 341-353.

JAMES, S. H. 1970. Complex hybridity in Isotoma petraea. II. Components and operation of a possible evolutionary mechanism. Heredity, 25, 53-78. 
JAMES, S. H. 1979. Chromosome numbers and genetic systems in the trigger plants of Western Australia (Stylidium; Stylidiaceae). Aust. J. Bot., 27, 17-25.

JAMES, S. H., SAMPSON, J. F. AND PLAYFORD, J. 1990. Complex hybridity in Isotoma petraea. VII. Assembly of the genetic system in the 06 Pigeon Rock population. Heredity, 64, 289-295.

JAMES, S. H., PLAYFORD, J. AND SAMPSON, J. F. 1991. Complex hybridity in Isotoma petraea. VIII. Variation for seed aborting lethal genes in the 06 Pigeon Rock population. Heredity, 66, 178-180.

KENDRICK, J. AND KNOX, R. B. 1989. Quantitative analysis of selfincompatibility in trees of seven species of Acacia. J. Hered., 80, 240-245.

LANDE, R. AND SCHEMSKE, D. W. 1985. The evolution of selffertilization and inbreeding depression in plants. I. Genetic models. Evolution, 39, 24-40.

LEDIG, F. T. 1986. Heterozygosity, heterosis, and fitness in outbreeding plants. In: Soule, M. E. (ed.) Conservation Biology: the Science of Scarcity and Diversity. Sinauer, Sunderland, MA, pp 77-104.

MAYNARD SMITH, J. 1977. Why the genome does not congeal. Nature, 268, 693-696.

PEAKALL, R. AND JAMES, S. H. 1989. Outcrossing in an ant pollinated clonal orchid. Heredity, 62, 161-167.
SAMPSON, J. F., HOPPER, S. D. AND JAMES, S. H. 1989. The mating system and population genetic structure in a bird pollinated mallee, Eucalyptus rhodantha. Heredity, 63, 383-393.

SORENSEN, F. E. 1969. Embryonic genetic load in coastal Douglas-fir, Pseudotsuga menziesii var. menziesii. Am. Nat. 103, 389-398.

SMITH-WHITE, s. 1948. Polarised segregation in a stable triploid. Heredity, 2, 119-129.

SMITH-WHITE, s. 1955. The life history and genetic system of Leucopogon juniperinus. Heredity, 9, 79-91.

SMITH-whITE, s. 1959. Pollen development patterns in the Epacridaceae. A problem in cytoplasm-nucleus interaction. Proc. Linn Soc. N.S. W., 84, 8-35.

TEMPLETON, A. R. 1980a. The theory of speciation via the founder principle. Genetics, 91, 1011-1038.

TEMPLETON, A. R. 1980 b. Modes of speciation and inferences based on genetic distances. Evolution, 34, 719-729.

TURNER, J. R. G. 1967. Why does the genotype not congeal? Evolution, 21, 645-656.

WeinS, D., CALVIN, C., WILSON, C. A., FRANK, D. AND LEAVEY, s. 1987. Reproductive success, spontaneous embryo abortion, and genetic load in flowering plants. Oecologia, 71, 501-509. 